\title{
A CLASS OF REGULAR MATRICES
}

\author{
GODFREY L. ISAACS
}

\begin{abstract}
Let $m$ be the space of real, bounded sequences $x=\left\{x_{k}\right\}$ with the sup norm, and let $A=\left(a_{n, k}\right)$ be a regular (i.e., Toeplitz) matrix. We consider the following two possible conditions for $A$ : (1) $\sum_{k=1}^{\infty}\left|a_{n, k}\right| \rightarrow 1$ as $n \rightarrow \infty$, (2) $\sum_{k=1}^{\infty}\left|a_{n, k}-a_{n, k+1}\right| \rightarrow 0$ as $n \rightarrow \infty$. G. Das [J. London Math. Soc. (2) 7 (1974), 501-507] proved that if a regular matrix $A$ satisfies both (1) and (2) then (3) $\varlimsup_{n \rightarrow \infty}(A x)_{n}<q(x)$ for all $x \in m$, where $q(x)$ $=\inf _{n_{i}, p} \varlimsup_{k \rightarrow \infty} p^{-1} \sum_{i=1}^{p} x_{n_{i}+k}$. Das used "Banach limits" and HahnBanach techniques, and stated that he thought it would be "difficult to establish the result . . . by direct method". In the present paper an elementary proof of the result is given, and it is shown also that the converse holds, i.e., for a regular $A$, (3) implies (1) and (2). Hence (3) completely characterizes the class of regular matrices satisfying (1) and (2).
\end{abstract}

1. We write $m$ for the space of real, bounded sequences $x=\left\{x_{k}\right\}$ with the sup norm, and we say that an infinite, real matrix $A=\left(a_{n, k}\right)$ is regular iff $\lim _{n \rightarrow \infty}(A x)_{n}=c$ when $\lim _{n \rightarrow \infty} x_{n}=c$. It is known (see [2, p. 502]) that $A$ is regular iff (a) $\sup _{n} \sum_{k=1}^{\infty}\left|a_{n, k}\right|<\infty$, (b) $\lim _{n \rightarrow \infty} \sum_{k=1}^{\infty} a_{n, k}=1$, (c) $\lim _{n \rightarrow \infty} a_{n, k}=$ $0 \forall k$. From (a) it is clear that $A x \in m$ for all $x \in m$.

We shall be concerned with two classes of regular matrices: the one consists of those regular matrices $A=\left(a_{n, k}\right)$ for which $\left(\left|a_{n, k}\right|\right)$ is regular. From the conditions above this happens iff

$$
\lim _{n \rightarrow \infty} \sum_{k=1}^{\infty}\left|a_{n, k}\right|=1 .
$$

The other class consists of those regular matrices $A$ whose action on an $x \in m$ is asymptotically independent of a "shift" in $x$, i.e., which satisfy $\lim _{n \rightarrow \infty}(A(x-D x))_{n}=0$ for all $x \in m$, where, if $x=\left\{x_{k}\right\}, D x=\left\{x_{k+1}\right\}$. By Lemma 3 below this happens iff

$$
\lim _{n \rightarrow \infty} \sum_{k=1}^{\infty}\left|a_{n, k}-a_{n, k+1}\right|=0 .
$$

We shall prove

THEOREM 1. For a regular matrix $A$ the following are equivalent:

(i) A satisfies (1) and (2);

$$
\varlimsup_{n \rightarrow \infty}(A x)_{n} \leqslant q(x) \text { for all } x \in m,
$$

Presented to the Society, August 23, 1974; received by the editors January 28, 1975. AMS (MOS) subject classifications (1970). Primary 40C05, 40H05; Secondary 46B 15.

Key words and phrases. Regular matrices, Toeplitz matrices, Banach limits, almost convergence. 
where

$$
q(x)=\inf _{n_{i}, p} \varlimsup_{k \rightarrow \infty} p^{-1} \sum_{i=1}^{p} x_{n_{i}+k} .
$$

The quantity $q(x)$ arises in connection with Banach limits [2, p. 501]: the Banach limits on $m$ are precisely the linear functionals $f$ on $m$ satisfying $f(x) \leqslant q(x)$ [2, Theorem 1]. From (3) we thus have that every $x \in m$ which is almost convergent (i.e., for which $-q(-x)=q(x)$ ) is summed by $A$ to $q(x)$, which in turn is the common value of all the Banach limits.

G. Das [2, Corollary to Theorem 2] proved that (i) implies (ii) by using Banach limits and Hahn-Banach techniques. He stated that he thought it would be "difficult to establish the result . . . by direct method". Our proof of Theorem 1 below uses only elementary methods.

2. Lemmas. Lemma 1 and its proof are substantially due to Lorentz [4, pp. $170-171]$.

LEMMA 1. If $x \in m$ then $q^{*}(x)=\varlimsup_{\lim _{K \rightarrow \infty}} K^{-1} \sum_{k=1}^{K} x_{k+n}$ is independent of $n$ and satisfies $q^{*}(x) \leqslant q(x)$ uniformly in $n$, i.e., given $\varepsilon>0$ there exists $k^{\prime}$ independent of $n$ such that

$$
K^{-1} \sum_{k=1}^{K} x_{k+n}<q(x)+\varepsilon \text { for all } K \geqslant k^{\prime} \text { and all } n \geqslant 0 \text {. }
$$

Proof. Taking two consequent values of $n$ in the expression for $q^{*}(x)$ and subtracting shows at once that $q^{*}(x)$ is independent of $n$. Now, writing $x_{k}=\left(x_{k}-b\right)+b$, where $b=\inf x_{k}$, it is sufficient for the rest to take $x_{k} \geqslant 0$ for all $k$. By the definition of $q(x)$ we see that given $\varepsilon>0$ there exist $n_{i}, p, k_{\varepsilon}\left(n_{i} \leqslant n_{i+1}\right)$ such that $p^{-1} \sum_{i=1}^{p} x_{n_{i}+k+n}<q(x)+(\varepsilon / 2)$ for all $k \geqslant k_{\varepsilon}$, and all $n \geqslant 0$. Write $k^{*}=\max \left(k_{\varepsilon}, n_{p}\right)$. For $K>k^{*}$ consider

$$
S=K^{-1} \sum_{k=1}^{K} p^{-1} \sum_{i=1}^{p} x_{n_{i}+k+n}
$$

Splitting the outer sum into sums over the ranges $1 \leqslant k \leqslant k^{*}$ and $k^{*}+1$ $\leqslant k \leqslant K$, we see that the first term obtained is $\leqslant\left(k^{*}\|x\|\right) / K<\varepsilon / 4$ for $K>$ some $k_{1}$, where $k_{1}$ is independent of $n \geqslant 0$, and the second is $\leqslant q(x)+$ $(\varepsilon / 2)$.

Rewriting $S$ as $(K p)^{-1} \sum_{i=1}^{p} \sum_{k=1}^{K} x_{n_{i}+k+n}$ and then splitting the inner sum into sums having respective ranges $1 \leqslant k \leqslant n_{p}-n_{i}, n_{p}-n_{i}+1 \leqslant k \leqslant K-$ $n_{i}+n_{1}, K-n_{i}+n_{1}+1 \leqslant k \leqslant K$, we see that with $S=A+B+C$,

$$
0 \leqslant A+C \leqslant\left(2 n_{p} p\|x\| / p\right) K^{-1}<\varepsilon / 8 \text { for all } K>\text { some } k_{2}
$$

where $k_{2}$ is independent of $n \geqslant 0$, and

$$
\begin{aligned}
0 & \leqslant B=K^{-1} \sum_{r=n_{p}+1}^{n_{1}+K} x_{r+n}=K^{-1}\left\{\sum_{r=1}^{K}-\sum_{r=1}^{n_{p}}+\sum_{r=K+1}^{K+n_{1}}\right\} x_{r+n}, \\
& =T-U+V,
\end{aligned}
$$

say. Clearly, $U$ and $V$ are each nonnegative and $\leqslant\left(\|x\| n_{p}\right) / K<\varepsilon / 16$ for all $K>$ some $k_{3}$, where $k_{3}$ is independent of $n \geqslant 0$; hence 


$$
\begin{aligned}
0 & \leqslant T=B+U-V=S-A-C+U-V \\
& <q(x)+(3 \varepsilon / 4)+(\varepsilon / 8)+(\varepsilon / 8)=q(x)+\varepsilon
\end{aligned}
$$

for all $K>k^{\prime}$, where $k^{\prime}=\max \left(k^{*}, k_{1}, k_{2}, k_{3}\right), k^{\prime}$ being independent of $n \geqslant 0$. This gives (4).

LEMMA 2. For a regular matrix $A$ the following are equivalent:

(i) $A$ satisfies (1);

(ii) $\overline{\lim }_{n \rightarrow \infty}(A x)_{n} \leqslant \overline{\lim }_{n \rightarrow \infty} x_{n}$ for all $x \in m$.

This is given in [2, p. 503] $\left(\tau^{+}\right.$in (2.4) should read $\left.\tau\right)$. An elementary proof is given in [1, pp. 150-152].

LEMMA 3. For a regular matrix $A,(2)$ is true iff

$$
\lim _{n \rightarrow \infty}(A(x-D x))_{n}=0 \text { for all } x \in m,
$$

where, if $x=\left\{x_{k}\right\}, D x=\left\{x_{k+1}\right\}$.

This is given in $[2, \mathrm{p} .502]$. If we write $(5)$ as $\lim _{n \rightarrow \infty}((A-A D) x)_{n}=0$ for all $x \in m$, so that $A$ and $A D$ are "absolutely equivalent", the result is covered by the elementary proof in [1, pp. 105-107].

3. Proof of Theorem 1: (i) $\Rightarrow$ (ii). As in the proof of Lemma 1, it is sufficient to take $x_{k} \geqslant 0$ for all $k$. Now by the convergence of the series in (1),

$$
(A x)_{n}=\sum_{k=1}^{\infty} a_{n, k} x_{k}=\sum_{t=0}^{\infty} \sum_{k=t k^{\prime}+1}^{t k^{\prime}+k^{\prime}} a_{n, k} x_{k} \text { for all } x \in m,
$$

where, for given $\varepsilon>0, k^{\prime}$ satisfies (4). Now by writing $x_{k}=s_{k}-s_{k-1}$, where

$$
s_{k}= \begin{cases}\sum_{k=v}^{k} x_{r}, & k \geqslant v, \\ 0, & k<v,\end{cases}
$$

we have (cf. [3, line 1, p. 437])

$$
\sum_{k=v}^{w} a_{n, k} x_{k}=\sum_{k=v}^{w-1} s_{k}\left(a_{n, k}-a_{n, k+1}\right)+s_{w} a_{n, w}=E+F,
$$

say. We apply this with $v=t k^{\prime}+1, w=t k^{\prime}+k^{\prime}$; since

$$
0 \leqslant s_{k} \leqslant s_{w}=\sum_{p=1}^{k^{\prime}} x_{p+t k^{\prime}}<k^{\prime}(q(x)+\varepsilon) \text { for all } t \geqslant 0,
$$

we obtain

$$
E+F \leqslant k^{\prime}(q(x)+\varepsilon)\left(\sum_{k=t k^{\prime}+1}^{t k^{\prime}+k^{\prime}}\left|a_{n, k}-a_{n, k+1}\right|+\left|a_{n, t k^{\prime}+k^{\prime}}\right|\right) .
$$

Now

$$
\begin{aligned}
k^{\prime}\left|a_{n, t k^{\prime}+k^{\prime}}\right| & =\sum_{s=2}^{k^{\prime}+1}\left|a_{n, t k^{\prime}+k^{\prime}}\right| \\
& =\sum_{s=2}^{k^{\prime}+1}\left(\left|a_{n, t k^{\prime}+s-1}\right|+\sum_{r=t k^{\prime}+s}^{t k^{\prime}+k^{\prime}}\left(\left|a_{n, r}\right|-\left|a_{n, r-1}\right|\right)\right) .
\end{aligned}
$$


Writing $r=t k^{\prime}+s-1$ in the first sum, and majorizing $\left|a_{n, r}\right|-\left|a_{n, r-1}\right|$ by $\left|a_{n, r}-a_{n, r-1}\right|$ and then replacing $r=t k^{\prime}+s$ by $r=t k^{\prime}+2$ in the second sum, we obtain, by (6)-(8),

$$
(A x)_{n} \leqslant(q(x)+\varepsilon)\left(\sum_{r=1}^{\infty}\left|a_{n, r}\right|+2 k^{\prime} \sum_{r=2}^{\infty}\left|a_{n, r}-a_{n, r-1}\right|\right)
$$

for all $n \geqslant 0, k^{\prime}$ being independent of $n$. Hence

$$
\varlimsup_{n \rightarrow \infty}(A x)_{n} \leqslant(q(x)+\varepsilon)\left(1+2 k^{\prime} \cdot 0\right)=q(x)+\varepsilon .
$$

Since $\varepsilon$ is arbitrarily small, we obtain (3).

(ii) $\Rightarrow$ (i). (1) follows at once by Lemma 2 and the fact that (by taking $\left.n_{i}=p=1\right) q(x) \leqslant \varlimsup_{n \rightarrow \infty} x_{n}$. Now as in [2, p. 502],

$$
\begin{aligned}
\varlimsup_{n \rightarrow \infty}(A(x-D x))_{n} & \leqslant q(x-D x) \leqslant \varlimsup_{k \rightarrow \infty} p^{-1} \sum_{i=1}^{p}\left(x_{i+k}-x_{i+k+1}\right) \text { for all } p, \\
& \leqslant \varlimsup_{k \rightarrow \infty} 2\|x\| / p .
\end{aligned}
$$

Since $p$ is arbitrarily large, $\varlimsup_{n \rightarrow \infty}(A(x-D x))_{n} \leqslant 0$. Replacing $x-D x$ by $D x-x$ we get $\varlimsup_{n \rightarrow \infty}(A(D x-x))_{n} \leqslant 0$, i.e. $\underline{\lim }_{n \rightarrow \infty}(A(x-D x))_{n} \geqslant 0$. This gives (5), and hence (2), by Lemma 3.

\section{REFERENCES}

1. R. G. Cooke, Infinite matrices and sequence spaces, Dover, New York, 1955.

2. G. Das, Banach and other limits, J. London Math. Soc. (2) 7 (1974), 501-507. MR 49 \# 924.

3. G. L. Isaacs, An iteration formula for fractional differences, Proc. London Math. Soc. (3) 13 (1963), 430-460. MR 27 \#5061.

4. G. G. Lorentz, A contribution to the theory of divergent sequences, Acta Math. 80 (1948), 167-190. MR 10, 367.

Department of Mathematics, Lehman College, City University of New York, Bronx, NEW YORK 10468 